\title{
Application of Fixed Point Theorem and Error Bounds
}

\author{
S. A. M. Mohsenialhosseini \\ Faculty of Mathematics, Vali-e-Asr University of Rafsanjan, Rafsanjan, Iran \\ Email: amah@vru.ac.ir, mohsenialhosseini@gmail.com
}

Received 5 April 2014; revised 15 May 2014; accepted 25 June 2014

Copyright (C) 2014 by authors and OALib.

This work is licensed under the Creative Commons Attribution International License (CC BY). http://creativecommons.org/licenses/by/4.0/

\section{(c) (i) Open Access}

\begin{abstract}
This paper is introduced as a survey of the results on some generalization of Banach's fixed point, their approximations to the fixed point and error bounds, and also contains some new fixed point theorems and applications.
\end{abstract}

\section{Keywords}

Fixed Point Theorems, Error Bounds, Linear Algebraic Equations, Nonlinear Equation

Subject Areas: Functional Analysis, Numerical Mathematics

\section{Introduction}

An interesting generalization of the Banach contraction principle is given by Boyed and Wong [1] and non-archimedean version of the results in this subject has been recently studied by De Kimpe and Siddiqui [2].

The Fixed points have long been used in analysis to solve various kinds of partial differential equations, ordinary differential equations and integral equations [3]. Cauchy (1789-1857) did fundamental work on existence theorems in differential equations. Fixed point theorems of ordered Bananch spaces provide us exact or approximate solutions of boundary-value problems. For details, one can refer to Amann [4], Collatz [5] and Franklin [6]. We did works on Theorems concerning the existence and properties that are known as approximate best proximity theorems [7], and approximate best proximity existence theorems in approximation differential equations [3].

Definition 1.1. Let $X$ be a set and $T: X \rightarrow X$ be a mapping. A fixed point of the mapping $T$ is a point $x \in X$ such that $T x=x$. In other words, a fixed point of $T$ is a solution of the functional equation $T x=x$ for $x \in X$.

Definition 1.2. Let $T$ be a mapping of a metric space $X$ into itself then $T$ is called a ontraction mapping if there exists a constant $L, \quad 0 \leq L<1$, such that $d(T(x), T(y)) \leq L d(x, y)$ for all $x, y \in X$.

Theorem 1.3. [8] Every contraction mapping $T: X \rightarrow X$ defined on a complete metric space $X$ into itself 
has a unique fixed point $u \in X$. Moreover, if $x_{0}$ is any point in $X$ and the sequence $x_{n}$ is defined by

$$
x_{1}=T\left(x_{0}\right), x_{2}=T\left(x_{1}\right), \cdots, x_{n}=T\left(x_{n-1}\right),
$$

Then $\lim _{n \rightarrow \infty} x_{n}=u$ and

$$
d\left(x_{n}, u\right) \leq \frac{L^{n}}{(1-L)} d\left(x_{1}, x_{0}\right)
$$

and that $L$ is as in Definition 1.2.

Corollary 1.4. [8] Let $L(X, d)$ be a complete metric space and $T: X \rightarrow X$ be a contraction on a closed ball $B\left(x_{0}, r\right)=\left\{x: d\left(x, x_{0}\right) \leq r\right\}$. Moreover, assume that $d\left(x_{0}, T x_{0}\right) \leq(1-L) r$. Then the iterative sequence (1) converges to an $u \in B$. This is a fixed point of $T$ and is the only fixed point of $T$ in $B$.

\section{Some Results on Fixed Point}

In this section, we give some result on fixed point in complete metric space and its applications.

Theorem 2.1. If $X$ is a complete metric space, and let $T: X \rightarrow X$ be a map such that $T^{r}$ is contraction for some integer $r>0$. Then for every $n \in N$ and $y \in X$,

$$
\lim _{n \rightarrow \infty} T^{r_{n}}(y)=u \text {. }
$$

Proof: Since $T^{r}$, where $r$ is a positive integer, is a contraction mapping by Theorem 1.3, there exists an unique fixed point $u$ of $T^{r}$ i.e. $T^{r}(u)=u$. Now, let $S=T^{r}$, therefore, $S(u)=u$. This implies that

$$
\begin{aligned}
& S^{2}(u)=S(S(u))=S(u)=u, \\
& S^{3}(u)=S^{2}(S(u))=S^{2}(u)=u, \\
& S^{n}(u)=S^{n-1}(S(u))=S^{n-1}(u)=\cdots=u,
\end{aligned}
$$

and so $T(u)=T\left(S^{n}(u)\right)=S^{n}(T(u))=S^{n} y$. Therefore for every $n \geq 1$ and $y \in X$,

$$
\begin{aligned}
d\left(S^{n}(y), u\right) & =d\left(S^{n}(y), S^{n}(u)\right) \leq c d\left(S^{n-1}(y), S^{n-1}(u)\right) \\
& \leq c^{2} d\left(S^{n-2}(y), S^{n-2}(u)\right) \leq \cdots \leq c^{n} d(y, u) .
\end{aligned}
$$

It follows that

$$
\lim _{n \rightarrow \infty} d\left(S^{r_{n}}(y), u\right)=0,
$$

since $c^{n} \rightarrow 0$. thus, $\lim _{n \rightarrow \infty} S^{n}(y)=u$. $\lim _{n \rightarrow \infty} T^{r_{n}}(y)=u$.

Theorem 2.2. Let $L(X, d)$ be a complete metric space and let $T: X \rightarrow X$ and $S: X \rightarrow X$ be two maps contraction. If for every $x \in X$, chosen suitably. Then for every $x \in X$,

$$
d\left(T^{m} x, S^{m} y\right) \leq \lambda \frac{1-L^{m}}{1-L}, \quad m=1,2, \cdots
$$

and that $L$ is as in Definition 1.2.

Proof: The relation is true for $m=1$. We use the principle of induction in order to prove this relation. Let it be true for all $m \geq 1$. Then,

$$
\begin{aligned}
d\left(T^{m+1}(x), S^{m+1}(x)\right) & =d\left(T T^{m}(x), S S^{m}(x)\right) \\
& \leq d\left(T T^{m}(x), T S^{m}(x)\right)+d\left(T S^{m}(x), S S^{m}(x)\right) \\
& \leq L d\left(T^{m}(x), S^{m}(x)\right)+\lambda \leq L \lambda \frac{1-L^{m}}{1-L}+\lambda \\
& =\frac{L \lambda-L^{m+1} \lambda+\lambda-L \lambda}{1-L}=\lambda \frac{1-k^{m+1}}{1-k}
\end{aligned}
$$

Thus, the relation is true for $m+1$. 
Corollary 2.3. Let $L(X, d)$ be a complete metric space and let $T: X \rightarrow X$ be a contraction on $X$. Moreover, the iteration sequence

$$
x_{1}=T\left(x_{0}\right), x_{2}=T\left(x_{1}\right)=T^{2}\left(x_{0}\right), \cdots, x_{n}=T^{n}\left(x_{0}\right)
$$

with arbitrary $x_{0} \in X$ converges to the unique fixed point $u$ of $T$. Error estimate is the following estimate prior estimate:

$$
d\left(x_{m}, u\right) \leq \frac{L^{m}}{1-L} d\left(x_{0}, x_{1}\right), \quad m=1,2, \cdots
$$

Proof: The First statement is obvious by

$$
\begin{aligned}
d\left(x_{m}, x_{n}\right) & \leq d\left(x_{m}, x_{m+1}\right)+d\left(x_{m+1}, x_{m+2}\right)+\cdots+d\left(x_{n-1}, x_{n}\right) \\
& \leq\left(L^{m}+L^{m+1}+\cdots+L^{n-1}\right) d\left(x_{0}, x_{1}\right)
\end{aligned}
$$

thus

$$
d\left(x_{m}, x_{n}\right) \leq \frac{L^{m}}{1-L} d\left(x_{0}, x_{1}\right), \quad(n>m) .
$$

Now, inequality (3) follows from (4) by letting $n \rightarrow \infty$. We have

$$
d\left(x_{m}, x_{n}\right) \leq \frac{L^{m}}{1-L} d\left(x_{0}, x_{1}\right) .
$$

Corollary 2.4. Let $L(X, d)$ be a complete metric space and $T: X \rightarrow X$ be a contraction on a closed ball $B\left(x_{0}, r\right)=\left\{x: d\left(x, x_{0}\right) \leq r\right\}$. Moreover, assume that $d\left(x_{0}, T x_{0}\right) \leq(1-L) r$. Then prior error estimate is the following estimate:

$$
d\left(x_{m}, u\right) \leq L^{m} r .
$$

Proof: By Corollary (2.3) the iteration sequence is converges to the unique fixed point $u$ of $T$, and

$$
d\left(x_{m}, x_{n}\right) \leq \frac{L^{m}}{1-L} d\left(x_{0}, x_{1}\right),
$$

since $x_{1}=T x_{0}$ and $d\left(x_{0}, T x_{0}\right) \leq(1-L) r$, we have

$$
\begin{aligned}
d\left(x_{m}, u\right) & \leq \frac{L^{m}}{1-L} d\left(x_{0}, x_{1}\right)=\frac{L^{m}}{1-L} d\left(x_{0}, T x_{0}\right) \\
& <\frac{L^{m}}{1-L}(1-L) r=L^{m} r .
\end{aligned}
$$

Therefore $d\left(x_{m}, u\right) \leq L^{m} r$.

\section{Applications of Banach Contraction Principle on Complete Metric Space}

In this section, we apply Theorem 1.3 to prove existence of the solutions a system of $n$ linear algebraic equations with $n$ unknowns, and we show that applied of Corollary 2.3 and 2.4 in numerical analysis.

Application 3.1. Suppose we want to find the solution of a system of $n$ linear algebraic equations with $n$ unknowns:

$$
\left\{\begin{array}{c}
a_{11} x_{1}+a_{12} x_{2}+\cdots+a_{1 n} x_{n}=b_{1} \\
a_{21} x_{1}+a_{22} x_{2}+\cdots+a_{2 n} x_{n}=b_{2} \\
\vdots \\
a_{n 1} x_{1}+a_{n 2} x_{2}+\cdots+a_{n n} x_{n}=b_{n}
\end{array}\right.
$$

This system can be written as: 


$$
\left\{\begin{array}{c}
x_{1}=\left(1-a_{11}\right) x_{1}-a_{12} x_{2}-\cdots-a_{1 n} x_{n}+b_{1} \\
x_{2}=-a_{21} x_{1}+\left(1-a_{22}\right) x_{2}-\cdots-a_{2 n} x_{n}+b_{2} \\
\vdots \\
x_{n}=-a_{n 1} x_{1}-a_{n 2} x_{2}-\cdots+\left(1-a_{n n}\right) x_{n}+b_{n}
\end{array}\right.
$$

By assuming $\alpha_{i k}=a_{i j}+\delta_{i j}$, where

$$
\delta_{i j}= \begin{cases}0 & i \neq j \\ 1 & i=j\end{cases}
$$

Equation (6) can be written in the following equivalent form.

$$
x_{i}=\sum_{j=1}^{n} \alpha_{i j} x_{j}+b_{i} \quad i=1,2,3, \cdots, n
$$

If $x=\left(x_{1}, x_{2}, \cdots, x_{n}\right) \in R^{n}$ then Equation (7) can be written in the form $T(x)=x$, where $T: R^{n} \rightarrow R^{n}$ is defined by

$$
\text { Tx }=y \quad \text { where } y=\left(y_{1}, y_{2}, \cdots, y_{n}\right)
$$

and

$$
y_{i}=\sum_{j=1}^{n} \alpha_{i j} x_{j}+b_{i} \quad i=1,2,3, \cdots, n
$$

Finding solutions of the system described by Equations (5) or (6) is thus equivalent to finding the fixed point of the operator equation, Equation (8). In order to find a unique solution of $T$, i.e. a unique solution of Equation (8), we apply Theorem (1.3). In fact, we prove the following result. Equation (5) has a unique solution, if

$$
\sum_{j=1}^{n}\left|\alpha_{i j}\right|=\sum_{j=1}^{n}\left|a_{i j}+\delta_{i j}\right| \leq L<1, \quad i=1,2,3, \cdots, n
$$

For $x=\left(x_{1}, x_{2}, \cdots, x_{n}\right)$ and $x^{\prime}=\left(x_{1}^{\prime}, x_{2}^{\prime}, \cdots, x_{n}^{\prime}\right)$, we have

$$
d\left(T x, T x^{\prime}\right)=d\left(y, y^{\prime}\right)
$$

where

$$
\begin{gathered}
y=\left(y_{1}, y_{2}, \cdots, y_{n}\right) \in R^{n} \\
y=\left(y_{1}^{\prime}, y_{2}^{\prime}, \cdots, y_{n}^{\prime}\right) \in R^{n} \\
y_{i}=\sum_{j=1}^{n} \alpha_{i j} x_{j}+b_{i} \\
y_{i}^{\prime}=\sum_{j=1}^{n} \alpha_{i j} x_{j}^{\prime}+b_{i} \quad i=1,2,3, \cdots, n
\end{gathered}
$$

If $y=\left(y_{1}, y_{2}, \cdots, y_{n}\right) \in R^{n}$, then $d(y, y)=\operatorname{Sup}_{1 \leq i \leq n}\left|y_{i}\right|$. Therefore,

$$
\begin{aligned}
d\left(T x, T x^{\prime}\right) & =\operatorname{Sup}_{1 \leq i \leq n}\left|y_{i}-y_{i}^{\prime}\right|=\operatorname{Sup}_{1 \leq i \leq n}\left|\sum_{j=1}^{n} \alpha_{i j} x_{j}+b_{i}-\right| \sum_{j=1}^{n} \alpha_{i j} x_{j}^{\prime}-b_{i}|| \\
& =\operatorname{Sup}_{1 \leq i \leq n}\left|\sum_{j=1}^{n} \alpha_{i j}\left(x_{j}-x_{j}^{\prime}\right)\right| \leq \operatorname{Sup}_{1 \leq i \leq n} \sum_{j=1}^{n}\left|\alpha_{i j}\right|\left|x_{j}-x_{j}^{\prime}\right| \\
& \leq \operatorname{Sup}_{1 \leq j \leq n}\left|x_{j}-x_{j}^{\prime}\right| \operatorname{Sup}_{1 \leq i \leq n} \sum_{j=1}^{n}\left|\alpha_{i j}\right| \leq \operatorname{Sup}_{1 \leq j \leq n}\left|x_{j}-x_{j}^{\prime}\right|
\end{aligned}
$$

Since $d\left(x, x^{\prime}\right)=\operatorname{Sup}_{1 \leq j \leq n}\left|x_{j}-x_{j}^{\prime}\right|$, we have $d\left(T x, T x^{\prime}\right) \leq L d\left(x, x^{\prime}\right), 0 \leq L<1$, i.e. $T$ is a contraction mapping of the complete metric space $R^{n}$ into itself. Hence, by Theorem 1.3, there exists a unique fixed point $u$ 
of $T$ in $R^{n}$ i.e. $u$ is a unique solution of Equation (5).

Theorem 3.2. If $f(t)$ be a nonlinear integral equation as following:

$$
f(t)=\int_{0}^{t} \mathrm{e}^{-v t} \cos (p f(t)) \mathrm{d} v, \quad 0 \leq t \leq 1 ; 0<p<1
$$

Then it has a unique solution.

Proof: We apply the Theorem 1.3, we can prove that this equation has a unique continuous real-valued solution $f(t)$. Let $X=C[0,1]$, and the mapping $T: X \rightarrow X$ defined by $T(f)=f$ for $f \in X$, where $X$ is a complete metric space with $\sup d(x, y)$, is a contraction mapping:

$$
\cos (p a)-\cos (p b)=p(b-a) \sin \alpha
$$

where $\alpha$ lies between $p a$ and $p b$. Therefore, $|\cos (p a)-\cos (p b)| \leq p|b-a|$.

For functions $a(t)$ and $b(t)$; we get

$$
|\cos p a(t)-\cos p b(t)| \leq \sup _{0 \leq t \leq 1}|a(t)-b(t)|=d(a, b)
$$

For $f=T f$ and $g=T g$, we have

$$
\begin{aligned}
|T f-T g| & =\left|\int_{0}^{t} \mathrm{e}^{-v t}\right| \cos (p f(v))-\cos (p g(v))|d v| \\
& \leq \int_{0}^{t} \mathrm{e}^{-v t}|\cos (p f(v))-\cos (p g(v))| \mathrm{d} v \\
& \leq p d(f, g) \int_{0}^{t} \mathrm{e}^{-v t} \mathrm{~d} v \leq p d(f, g)
\end{aligned}
$$

Taking sup over $0 \leq t \leq 1$, we get

$$
\sup _{t}|T f(t)-T g(t)| \leq p d(f, g)
$$

or

$$
d(T(f), T(g)) \leq p d(f, g) .
$$

Theorem 3.3. Let $x_{0}$ be an initial value and the iterative sequence $\left\{x_{n}\right\}$ as following:

$$
x_{n}=g\left(x_{n-1}\right) \quad n=1,2, \cdots
$$

If $g$ is continuously differentiable on some interval $k=\left[x_{0}-r, x_{0}+r\right]$ and satisfies $\left|g^{\prime}(x)\right| \leq L<1$ on $k$ as well as

$$
\left|g\left(x_{0}\right)-x_{0}\right|<(1-L) r .
$$

Then $x=g(x)$ has a unique solution $u$ on $k$, the iterative sequence $\left\{x_{m}\right\}$ converges to that solution, and one has the error estimates

$$
\left|x-x_{m}\right|<L^{m} r, \quad\left|x-x_{m}\right|<L r .
$$

Proof: Suppose that $d(x, g(x))=|x-g(x)|$ for $x \in k$. By the mean-value theorem and the given condition, $g(x)$ is a contraction mapping of the complete metric space $k$ into itself. Hence, by Corollary 1.4 there exists a unique fixed point $u$ of $g$ in $k$, i.e. $u$ is a unique solution of $x=g(x)$. Also, the iteration sequence $\left\{x_{m}\right\}$ converges to $u$. Moreover, by $d(x, g(x))=|x-g(x)|$ and Corollary 2.4 it has the prior error

$$
\left|x-x_{m}\right|<L^{m} r \text {, }
$$

and the posterior estimate

$$
\left|x-x_{m}\right|<L r
$$




\section{References}

[1] Boyed, D.W. and Wong, J.S.W. (1969) On Non-Linear Contraction. Proceedings of the American Mathematical Society, 20, 458-464.

[2] Kimpe, N.D. and Siddiqui, A.H. (Reprint) Fixed Point Theorems in Non-Archimedean, Functional Analysis.

[3] Mohsenalhosseini, S.A.M., Mazaheri, H., Dehghan, M.A. and Bagheshahi, M. (2011) Application of Approximate Best Proximity Pairs. General Mathematical Note $(G M N)$, 7, 59-65.

[4] Amann, H. (1976) Fixed Point Equations and Nonlinear Eigenvalue Problems in or 4'ed Banach Spaces. SIAM Review, 18, 620-709.

[5] Collatz, L. (1966) Functional Analysis and Numerical Mathematics. Academic Press, New York.

[6] Franklin, J. (1980) Methods of Mathematical Economics. Springer Verlag, New York.

[7] Mohsenalhosseini, S.A.M., Mazaheri, H. and Dehghan, M.A. (2011) Approximate Best Proximity Pairs in Metric Space. Abstract and Applied Analysis, 2011, Article ID: 596971.

[8] Siddiqi, A.H. (1986) Functional Analysis with Application. Tata McGraw-Hill Publishing Company Limited, New Delhi. 